\title{
Genetic defects in pediatric-onset adrenal insufficiency in Japan
}

\author{
Naoko Amano ${ }^{1,2}$, Satoshi Narumi ${ }^{1,3}$, Mie Hayashi ${ }^{1}$, Masaki Takagi ${ }^{1,4}$, \\ Kazuhide Imai ${ }^{5}$, Toshiro Nakamura6, Rumi Hachiya ${ }^{1,4}$, Goro Sasaki ${ }^{1,7}$, \\ Keiko Homma ${ }^{8}$, Tomohiro Ishii' ${ }^{1}$ and Tomonobu Hasegawa ${ }^{1}$
}

${ }^{1}$ Department of Pediatrics, Keio University School of Medicine, Tokyo, Japan, ${ }^{2}$ Department of Pediatrics, Tokyo Saiseikai Central Hospital, Tokyo, Japan, ${ }^{3}$ Department of Molecular Endocrinology, National Research Institute for Child Health and Development, Tokyo, Japan, ${ }^{4}$ Department of Endocrinology and Metabolism, Tokyo Metropolitan Children's Medical Center, Tokyo, Japan, ${ }^{5}$ Department of Pediatrics, Nishibeppu National Hospital, Oita, Japan, ${ }^{6}$ Department of Pediatrics, Kumamoto Chuo Hospital, Kumamoto, Japan, ${ }^{7}$ Department of Pediatrics, Tokyo Dental College Ichikawa General Hospital, Chiba, Japan, and ${ }^{8} \mathrm{Clinical}$ Laboratory, Keio University Hospital, Tokyo, Japan

\author{
Correspondence \\ should be addressed \\ to T Hasegawa \\ Email \\ thaseg@a6.keio.jp
}

\section{Abstract}

Context: Most patients with pediatric-onset primary adrenal insufficiency (PAI), such as 21-hydroxylase deficiency, can be diagnosed by measuring the urine or serum levels of steroid metabolites. However, the etiology is often difficult to determine in a subset of patients lacking characteristic biochemical findings.

Objective: To assess the frequency of genetic defects in Japanese children with biochemically uncharacterized PAI and characterize the phenotypes of mutation-carrying patients.

Methods: We enrolled 63 Japanese children (59 families) with biochemically uncharacterized PAl, and sequenced 12 PAl-associated genes. The pathogenicities of rare variants were assessed based on in silico analyses and structural modeling. We calculated the proportion of mutation-carrying patients according to demographic characteristics. Results: We identified genetic defects in 50 (85\%) families: STAR in 19, NROB1 in 18, SAMD9 in seven, AAAS in two, $N N T$ in two, MC2R in one and CDKN1C in one. NROB1 defects were identified in $78 \%$ of the male patients that received both glucocorticoid and mineralocorticoid replacement therapy and had normal male external genitalia. STAR defects were identified in $67 \%$ of female and $9 \%$ of male patients. Seven of the 19 patients with STAR defects developed PAI at age two or older, out of whom, five did not have mineralocorticoid deficiency.

Conclusions: Molecular testing elucidated the etiologies of most biochemically uncharacterized PAI patients. Genetic defects such as NROB1 defects are presumed based on phenotypes, while others with broad phenotypic variability, such as STAR defects, are difficult to diagnose. Molecular testing is a rational approach to diagnosis in biochemically uncharacterized PAI patients.

\section{Introduction}

Primary adrenal insufficiency (PAI) is a rare life-threatening condition caused by disruption of cortisol synthesis in the adrenal cortex despite high ACTH levels. A large proportion of patients with PAI have congenital adrenal hyperplasia $(\mathrm{CAH})$, most of which is caused by enzymatic defects in cortisol biosynthesis, such as deficiencies in 21-hydroxylase and 3 3 -hydroxysteroid dehydrogenase
() 2017 European Society of Endocrinology Printed in Great Britain
$(1,2)$. These enzymatic defects usually yield specific findings in the urine and serum steroid metabolites such as high serum $17 \alpha$-hydroxyprogesterone levels and can be diagnosed biochemically (3).

However, a minor subset of patients with PAI lacks these biochemical features, and it is often difficult to determine the etiology based on the clinical findings alone.

Published by Bioscientifica Ltd. 
Most of these biochemically uncharacterized PAI patients would be classified into four groups based on International Classification of Pediatric Endocrine Diagnosis (ICPED, www.icped.org). The first group is $\mathrm{CAH}$, which is caused by STAR or CYP11A1 defects $(4,5)$. The patients belonging to this group usually have PAI with complete female phenotype due to destruction of the initial step of steroid biosynthesis in adrenal and gonads. The second group is congenital adrenal hypoplasia, which is caused by mutations in NROB1 encoding DAX1 or NR5A1 encoding SF-1 $(6,7)$. The second group includes the syndromic PAI, such as IMAGe syndrome (caused by CDKN1C mutations) and SERKAL syndrome (caused by WNT4 mutations) (8, 9). The recently reported MIRAGE syndrome (caused by SAMD9 mutations) might also belong to the second group (10). IMAGe syndrome comprises intrauterine growth restriction, metaphyseal dysplasia, adrenal hypoplasia congenital and genital anomalies; SERKAL syndrome comprises 46,XX sex reversal with dysgenesis of the kidneys, adrenals and lungs; and MIRAGE syndrome comprises myelodysplasia, infection, restriction of growth, adrenal hypoplasia, genital phenotypes and enteropathy. The third group is familial glucocorticoid deficiency (FGD), which is predominantly isolated glucocorticoid insufficiency. This group is mainly composed of patients with ACTH resistance caused by MC2R and MRAP defects $(11,12)$. NNT, TXNRD2 and MCM4 have been reported as additional causative genes for this third group $(13,14$, 15). Triple A syndrome (caused by AAAS defects), which comprises achalasia, addisonianism (adrenal insufficiency) and alacrima, also belongs to the third group (16). The fourth group is adrenal destruction due to metabolic and autoimmune causes (AIRE, ABCD1, PEX and LIPA defects) $(17,18,19,20)$. Some recently identified novel causative genes and atypical phenotypes of these genetic defects have complicated the clinical approaches used to treat biochemically uncharacterized PAI patients.

There are few studies on the frequency of genetic defects in biochemically uncharacterized PAI patients. In 2015, Guran et al. conducted a genetic screening study targeting 95 non-syndromic pediatric patients having biochemically uncharacterized PAI in Turkey and revealed that $81 \%$ of the study subjects had genetic defects (21). On the other hand, Chan et al. also conducted genetic analyses targeting more than 300 FGD patients in the United Kingdom and diagnosed about $60 \%$ of them genetically (22). However, it is not clear whether these mutation frequencies can be generalized to other ethnic groups including Japanese.
In the present study, we conducted genetic analyses in 63 nonconsanguineous Japanese children with biochemically uncharacterized PAI. We estimated the frequency of genetic defects in our cohort and characterized the phenotypes of the mutationcarrying patients.

\section{Subjects and methods}

\section{Subjects}

We enrolled 63 patients belonging to 59 families with biochemically uncharacterized PAI in Japan from 2000 to 2015. The inclusion criteria were as follows: (i) age at diagnosis less than 15 years; (ii) clinical diagnosis of PAI based on symptoms (e.g. fatigue, hyperpigmentation, and vomiting) and laboratory examinations (i.e., low or normal serum cortisol levels with high plasma ACTH levels). We excluded the patients with any specific findings of urine or serum steroid metabolites suggesting enzymatic defects (i.e. 21-hydroxylase deficiency and $3 \beta$-hydroxysteroid dehydrogenase deficiency), and two patients with adrenoleukodystrophy.

We divided the subjects into two groups according to the onset age (infancy, <1 year; childhood, 1-15 years). Moreover, we also classified male patients into two groups according to the phenotypes of their external genitalia (normal or underdeveloped).

We obtained written informed consent for study participation from each patient or his/her parents. The Ethics Committee of Keio University School of Medicine approved the study. In the 63 patients, one patient with IMAGe syndrome and eight patients with MIRAGE syndrome were reported previously $(10,23)$.

\section{Molecular analysis}

We extracted genomic DNA from peripheral lymphocytes of the study subjects using a standard technique. We selected the following 12 genes associated with biochemically uncharacterized PAI: CYP11A1, STAR (associated with CAH); CDKN1C, NROB1, NR5A1 and SAMD9 (associated with congenital adrenal hypoplasia); AAAS, MCM4, MC2R, MRAP, NNT and TXNRD2 (associated with isolated or FGD). All coding exons and flanking introns of these 12 genes were analyzed using standard PCR-based sequencing, targeted next-generation sequencing or whole exome sequencing (Supplementary Table 1 , see section on supplementary data given at the 
end of this article). Regarding the PCR-based sequencing, the primer sequences and PCR conditions are available upon request. In 20 of 63 patients, we performed targeted next-generation sequencing using the panel of congenital endocrine disorders (160 genes), which was included all coding exons and flanking introns of the following 13 PAI-associated genes: CYP11A, STAR, NROB1, NR5A1, SAMD9, AAAS, MCM4, MC2R, MRAP, NNT, TXNRD2, GPX1 and WNT4. In 10 of 63 patients, we performed whole exome sequencing. The protocol of targeted nextgeneration sequencing and whole exome sequencing has been described previously $(10,24,25)$. We conducted array comparative genomic hybridization analyses in four male patients (patients 30, 32, 36 and 37) suspected of having a submicroscopic deletion due to negative PCR amplification of NROB1 (Agilent SurePrint G3 Human CGH Microarray Kit; Agilent Technologies). The deletion breakpoints of patients 36 and 37 were determined using deletion-specific PCR and sequencing (data not shown).

We regarded truncating variants (e.g., nonsense, frameshift and splice-site variants), gene deletions and previously reported mutations as pathogenic. As for rare missense variants with allele frequencies lower than 0.002 in ExAC (http://exac.broadinstitute.org/), dbSNP147 (http://www.ncbi.nlm.nih.gov/SNP/), NHLBI Exome Sequencing Project (http://evs.gs.washington.edu/EVS/) and Human Genetic Variation Database (HGVD, http:// www.hgvd.genome.med.kyoto-u.ac.jp), we predicted the pathogenicity by means of both SIFT (http://sift.jcvi. org/) and PolyPhen-2 (http://genetics.bwh.harvard.edu/ pph2/). We also analyzed the three-dimensional modeled structures of the identified rare missense variants using PDB atomic coordinate files (Dax-1 3F5C and STAR 3POL) and PyMOL, version 0.99 (http://www.pymol.org/). By means of the splice prediction tool, Human Splicing Finder (http://www.umd.be/HSF3/index.html), we predicted the effect on splicing of the $A A A S$ variant, c.1182-3C $>\mathrm{G}$, which was outside of the canonical $2 \mathrm{bp}$ splice site.

\section{Results}

\section{Subject characteristics}

The characteristics of the 59 probands (35 males, 24 females) are summarized in Table 1 , and the clinical features of all 63 patients are described in Supplementary Table 1. Ten patients had a family history of PAI or unexplained death in infancy/childhood. Six of the 10 patients were suspected to have X-linked recessive inheritance based on positive family history of male siblings or maternal
Table 1 Subject characteristics.

\begin{tabular}{|c|c|}
\hline Variable & \\
\hline Number of the probands & 59 \\
\hline Legal sex; male/female & $35 / 24$ \\
\hline Family history & 10 \\
\hline Potential inheritance; X-linked ${ }^{a}$ & 6 \\
\hline Others $^{\mathrm{b}}$ & 4 \\
\hline Consanguineous family & 0 \\
\hline Age at diagnosis; median (IQR) (years) & $0.0(0.0-2.0)$ \\
\hline Infancy/childhoodc & $42 / 17$ \\
\hline Mineralocorticoid replacement & 45 \\
\hline $\begin{array}{l}\text { External genitalia of male patients; normal/ } \\
\text { underdeveloped }\end{array}$ & $30 / 5$ \\
\hline $\begin{array}{l}\text { Complete female external genitalia of } 46, X Y \\
\text { patients }^{d}\end{array}$ & 5 \\
\hline Nonendocrine features & 13 \\
\hline
\end{tabular}

aThe potential X-linked inheritance includes male patients with family

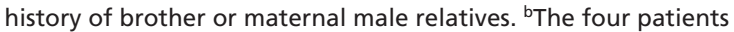
suspected to have other potential inheritance include three girls with a family history of siblings and a boy with a family history of a sister. 'The period of infancy is less than 1 year of age. ${ }^{d}$ The five $46, X Y$ patients with complete female external genitalia were assigned as female.

male relatives. Forty-two patients belonged to the infancy-onset group. Forty-five patients were suspected of having mineralocorticoid deficiency and received mineralocorticoid replacement therapy for salt-wasting symptoms or prolonged high plasma renin activity. Thirteen patients had nonendocrine features; pre- and postnatal growth restriction $(n=9)$, thrombocytopenia or anemia $(n=7)$, recurrent invasive infection $(n=7)$, chronic diarrhea $(n=5)$, congenital heart disease $(n=2)$, intrahepatic bile duct hypoplasia $(n=1)$, myelodysplastic syndrome $(n=1)$, metaphyseal dysplasia $(n=1)$, glycerol kinase deficiency $(n=1)$, Duchenne muscular dystrophy $(n=1)$ and apparent alacrima and achalasia $(n=1)$.

\section{Molecular analysis}

We identified genetic defects in 50 out of the 59 families (54 out of the 63 patients), including NROB1 defects in 18 families (20 patients), STAR defects in 19 families (19 patients), SAMD9 defects in seven families (eight patients), $A A A S$ defects in two families (three patients), $N N T$ defects in two families (two patients), MC2R defects in one family (one patient) and a CDKN1C defect in one family (one patient) (Fig. 1, Tables 2, 3 and Supplementary Table 1). We identified fourteen novel mutations; eight in NROB1, three in STAR, two in NNT and one in AAAS (Supplementary Table 2). Out of the fourteen mutations, four NROB1 mutations (p.Tyr130Profs*13, p.Gln283Leufs ${ }^{*}$, p.Gln318Alafs*71 and p.Gly402*), one STAR mutation (p.Asp246Argfs*37) and two NNT 


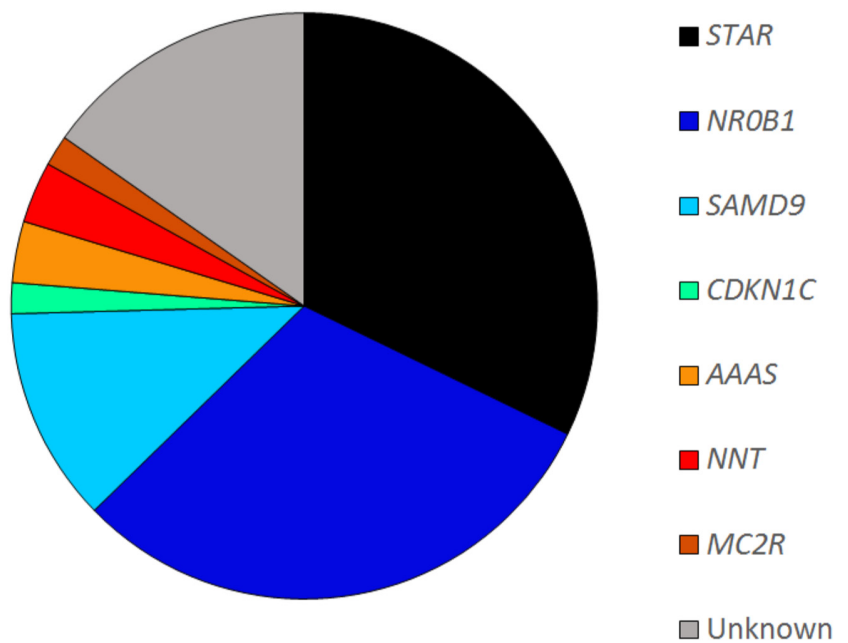

Figure 1

Proportions of genetic defects in our study cohort. Fifty of the 59 families harbored mutations in STAR $(n=19,32 \%)$, NROB1 $(n=18,31 \%)$, SAMD9 $(n=7,12 \%)$, AAAS $(n=2,3.4 \%), N N T$ $(n=2,3.4 \%), \operatorname{MC} 2 R(n=1,1.7 \%)$ and CDKN1C $(n=1,1.7 \%)$. A full colour version of this figure is available at http://dx.doi. org/10.1530/EJE-17-0027.

mutations (p.Gln40Lysfs*9 and p.Asp1001Valfs*6) were regarded as truncating. Five rare missense variants (NROB1; p.Arg288Pro, p.Leu294Gln and p.Leu381Phe, STAR; p.Thr164Lys and p.Arg188His) were predicted as deleterious by SIFT and as probably damaging by Polyphen-2, except for p.Arg188His predicted as possibly damaging. Moreover, these five missense variants were considered pathogenic by in silico structural modeling (Supplementary Fig. 1). The NROB1 variant, p.Asn430del was located in the ligand-like binding region, where previously reported missense mutations are clustered. And the $A A A S$ variant, c. $1182-3 \mathrm{C}>\mathrm{G}$, was predicted to create a new canonical acceptor site at c.1182-4_3 position, which was shifted by two base pairs. By array comparative genomic hybridization, we identified submicroscopic deletions in the four patients (patients 30, 32, 36 and 37) with negative PCR amplification of NROB1, accounting for $22 \%$ of the 18 NROB1 mutation-carrying patients. A nonsense STAR mutation, p.Gln258*, was observed in 20 (53\%) of 38 alleles in the 19 patients with STAR mutations (Supplementary Table 1).

\section{Proportions of families with mutations}

We identified genetic defects in 50 (85\%) out of the 59 families (Fig. 1). Eighteen (51\%) of the 35 male probands harbored NROB1 mutations, while 16 (67\%) of the 24 female probands harbored STAR mutations (Table 2). Six $(60 \%)$ of 10 probands with a family history harbored NROB1 mutations. Seven (54\%) of 13 probands with nonendocrine features harbored SAMD9 mutations.

\section{Phenotypes of the patients with mutations}

All 18 NROB1 mutation-carrying patients were treated with mineralocorticoid replacement therapy and had normal

Table 2 Family numbers with genetic defects according to demographic characteristics.

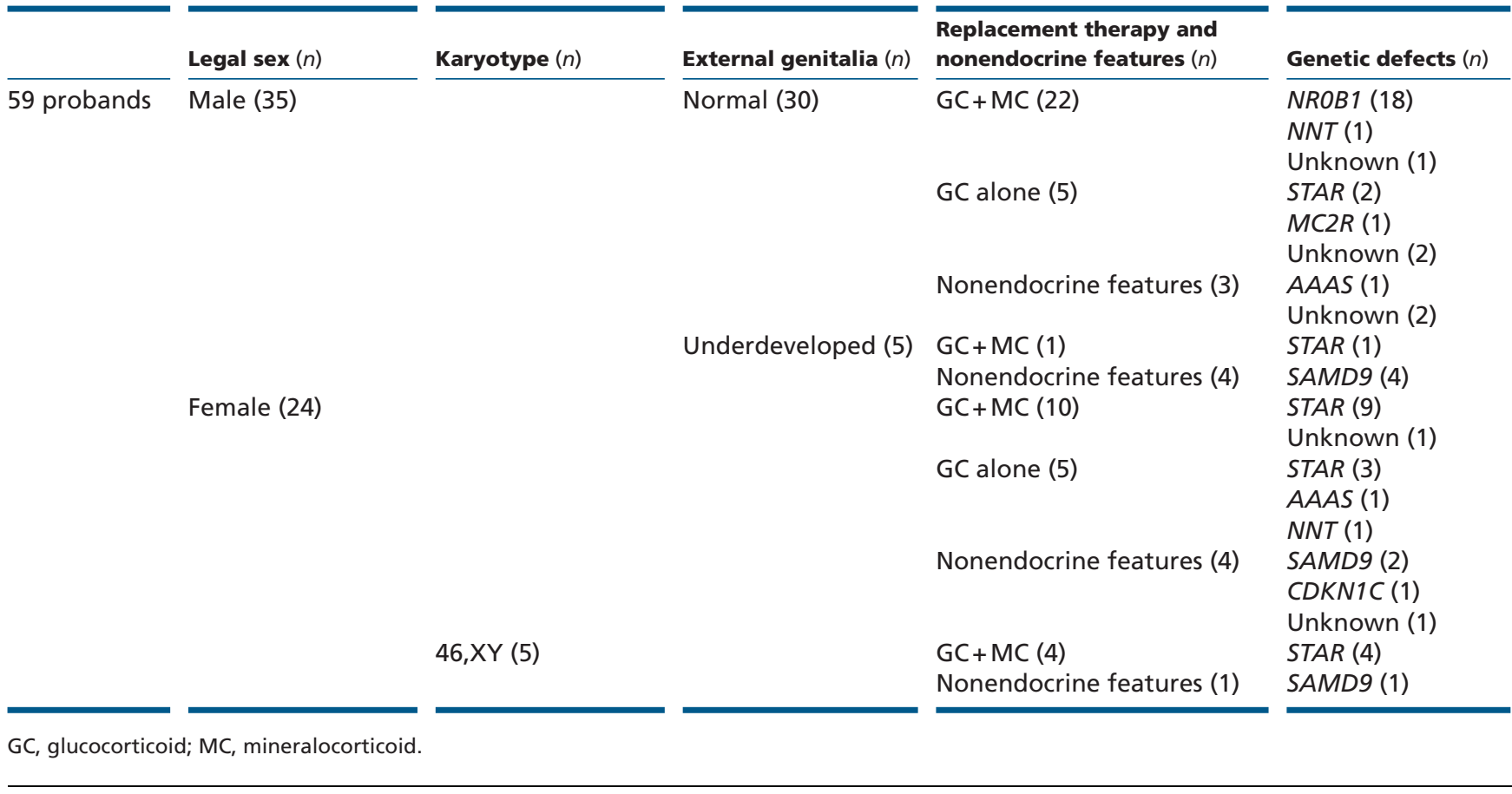


Table 3 Characteristics of the probands with genetic defects.

\begin{tabular}{l} 
Variable \\
\hline Number of probands \\
Legal sex; male/female \\
Family history \\
Potential inheritance; X-linked ${ }^{\mathrm{a}}$ \\
$\quad$ Others \\
Age at diagnosis; infancy/childhood \\
Mineralocorticoid replacement \\
External genitalia of male patients; normal/underdeveloped \\
Complete female external genitalia of $46, \mathrm{XY}$ patients \\
Nonendocrine features
\end{tabular}

\begin{tabular}{c}
\hline STAR \\
\hline 19 \\
$3 / 16$ \\
2 \\
0 \\
$2^{b}$ \\
$12 / 7$ \\
14 \\
$2 / 1$ \\
4 \\
0 \\
\hline
\end{tabular}

\begin{tabular}{c}
\hline NROB1 \\
\hline 18 \\
$18 / 0$ \\
6 \\
6 \\
0 \\
$13 / 5$ \\
18 \\
$18 / 0$ \\
0 \\
1 \\
\hline
\end{tabular}

\begin{tabular}{c}
\hline SAMD9 \\
\hline 7 \\
$4 / 3$ \\
1 \\
0 \\
1 \\
$7 / 0$ \\
5 \\
$0 / 4$ \\
1 \\
7 \\
\hline
\end{tabular}

\begin{tabular}{c}
\hline AAAS \\
\hline 2 \\
$0 / 2$ \\
1 \\
0 \\
$1^{c}$ \\
$0 / 2$ \\
0 \\
0 \\
0 \\
1 \\
\hline
\end{tabular}

\begin{tabular}{c}
\hline $\boldsymbol{N N T}$ \\
\hline 2 \\
$1 / 1$ \\
0 \\
0 \\
0 \\
$1 / 1$ \\
1 \\
$1 / 0$ \\
0 \\
0 \\
\hline
\end{tabular}

\begin{tabular}{|c|c|}
\hline MC2R & CDKN1C \\
\hline 1 & 1 \\
\hline $1 / 0$ & $0 / 1$ \\
\hline 0 & 0 \\
\hline 0 & 0 \\
\hline 0 & 0 \\
\hline $1 / 0$ & $1 / 0$ \\
\hline 0 & 1 \\
\hline $1 / 0$ & 0 \\
\hline 0 & 0 \\
\hline 0 & 1 \\
\hline
\end{tabular}

${ }^{a}$ The potential X-linked inheritance includes male patients with family history of brothers or maternal male relatives. ${ }^{b}$ The two patients were girls with a family history of a sibling. 'The patient was a boy with a family history of a sister. ${ }^{\mathrm{d}}$ The period of infancy is less than 1 year of age. ${ }^{\mathrm{e}}$ The $46, \mathrm{XY}$ patients with complete female external genitalia were assigned as female.

male external genitalia (Table 3 and Supplementary Table 3). Six NROB1 out of the 18 patients had a family history suggesting X-linked inheritance. The proportions of male patients with a family history, mineralocorticoid replacement therapy and normal male external genitalia were significantly higher in NROB1 defects group than those in non-NROB1 defects group. Eighteen (78\%) of the 23 male patients that had mineralocorticoid replacement therapy and normal external genitalia, carried NROB1 defects.

Of the 19 patients with STAR defects, there were three boys and 16 girls (Table 3 and Supplementary Table 1). Twelve patients (two boys and 10 girls, patients 1-11 and 16) were treated with mineralocorticoid replacement therapy and were diagnosed in infancy. The remaining seven (patients 12-15 and 17-19) developed PAI at 2 years of age or older (i.e nonclassic LCAH). In three (patients 12, 15 and 17) of them, the adrenal gland morphology was evaluated by ultrasonography or computed tomography and did not show enlargement despite high plasma ACTH levels. Six (patients 12-15, 18 and 19) of the seven nonclassic LCAH patients were compound heterozygous for the missense mutation p.Arg272Cys and a functionally null mutation (p.Gln258*, p.Thr44Hisfs*3 or c. $64+1 \mathrm{delG})$.
Two patients with NNT mutations were diagnosed around one year of age (Table 4). One patient (patient 47) with a homozygous frameshift mutation (p.Gln40Lysfs*9) was suspected of having mild mineralocorticoid deficiency because of high plasma renin activities (PRA: 8.6-45.4 ng/ $\mathrm{mL} / \mathrm{h}$, median $25.8 \mathrm{ng} / \mathrm{mL} / \mathrm{h}$, age-specific reference $\leq 15 \mathrm{ng} /$ $\mathrm{mL} / \mathrm{h}$ ) with normal serum sodium levels (136-140 mEq/L, median $139 \mathrm{mEq} / \mathrm{L}$ ) under hydrocortisone replacement therapy (Supplementary Note). The other patient (patient 48) had compound heterozygous NNT mutations (p.[Phe215Ser];[Asp1001Valfs*6]) and normal aldosterone production (PRA: $1.9-6.6 \mathrm{ng} / \mathrm{mL} / \mathrm{h}$, median: $2.8 \mathrm{ng} / \mathrm{mL} / \mathrm{h}$ ).

One patient (patient 45) with compound heterozygous AAAS mutations (c.1182-3C>G;p.[Phe215Ser]) showed apparent clinical signs suggestive of alacrima and achalasia. However, the other siblings (family 46) with compound heterozygous AAAS mutations (p.[Trp84*];[Arg342*]) were initially diagnosed as having 'ACTH resistance' (Supplementary Note). They had no clinical signs suggestive of triple A syndrome (i.e., achalasia, alacrima and neurologic disabilities). After identification of the $A A A S$ mutations, they were evaluated for achalasia, alacrima and neurologic disabilities for the first time. The older sister (patient 46-a) had mild dilatation of a hypokinetic esophagus and subclinical decreased tear

Table 4 Characteristics of NNT mutation-carrying patients.

Genotype
Age at diagnosis (months)
Serum Na (range, median, $\mathrm{mEq} / \mathrm{L}$ ) (sample number)
PRA (range, median, $\mathrm{ng} / \mathrm{mL} / \mathrm{h})^{\mathrm{a}}$ (sample number)
Administration of fludrocortisone

\begin{tabular}{c}
\hline Patient 40 \\
\hline p.[Q40fs];[Q40fs] \\
10 \\
136.0-140.2, $137.7(11)$ \\
$8.6-45.4,25.8(9)$ \\
Yes
\end{tabular}

\begin{tabular}{crr}
\hline Patient 41 & & $\boldsymbol{P}$ \\
\cline { 1 - 1 } p.[F215S];[D1001fs] & & \\
14 & & \\
$138.0-141.0,139.0(10)$ & & 0.20 \\
$1.9-6.6,2.8(10)$ & & $<0.05$ \\
No &
\end{tabular}

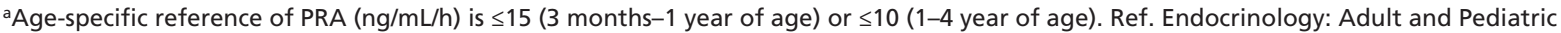
(seventh edition) 2016. pp. 2655-2688. 
production. The younger brother (patient 46-b) was found to have subclinical decreased tear production.

\section{Discussion}

In the present study, we identified genetic defects in $85 \%$ (95\% CI: 76-94\%) of Japanese patients with biochemically uncharacterized PAI. Previously, the proportion of genetic defects in biochemically uncharacterized PAI patients has been reported in Turkey (21). The proportion of genetic defects in our Japanese cohort was comparable to that of the Turkish cohort (81\%, 95\% CI: 73-90\%). However, the characteristics of study subjects substantially differed between these two studies. In our Japanese cohort, there was no case of consanguineous family, seven (30\%) out of the total 23 biallelic mutations were homozygous. Six (patients 1-5, and 11) of them harbored the STAR defect, p.Gln258*, while the remaining one (patient 47) harbored the NNT defect, p.Gln40Lysfs ${ }^{*} 9$. Contrastingly, in the Turkish cohort, $74 \%$ of families were consanguineous, and 98\% of the total biallelic mutations were homozygous. Despite this difference, the high frequencies of genetic defects in biochemically uncharacterized PAI were similar, regardless of the patients' ethnicities. On the other hand, the frequency of genetic defects was not so high in the United Kingdom (22) probably due to greater racial variation. Further studies are required to reproduce these high frequencies in other ethnic groups.

The relative proportions of genetic defects differed between our Japanese cohort and the Turkish cohort. In our Japanese study, the proportion of STAR defects reached $32 \%$, which was significantly higher than that in the Turkish cohort $(12 \% ; P=0.004$ by Fisher's exact test). The STAR mutation, p.Gln258*, accounted for most homozygous mutations in our cohort. This is likely due to the presence of this common STAR mutation, p.Gln258*, in China, Korea and Japan $(26,27,28)$. In addition, the proportions of $M C 2 R$, which was $26 \%$ in the Turkish cohort, differed from the findings of our study, with only one compound heterozygote of $M C 2 R$ mutations being observed in our Japanese cohort. This difference is likely due to the presence of a commonly detected mutation (p.Val187Alafs*29) in the Turkish study subjects. Moreover, CYP11A1 mutations, which accounted for $9.5 \%$ in the Turkish cohort, were not observed in our Japanese cohort. The relative proportions of causative genes in biochemically uncharacterized PAI must differ among ethnicities.
Eighteen $(51 \%)$ of the 35 male patients harbored NROB1 mutations. Moreover, 18 (78\%) of the 23 male PAI patients with mineralocorticoid replacement therapy and normal male external genitalia, and six boys who were suspected to have X-linked inheritance harbored NROB1 mutations. These results illuminate the importance of these factors (mineralocorticoid deficiency, external genitalia phenotype and family history) for clinical diagnosis of X-linked adrenal hypoplasia congenita. On the other hand, sixteen (67\%) of the 24 female patients harbored STAR mutations. These 16 patients included four $46, \mathrm{XY}$ neonates with complete female external genitalia. These four patients contribute the majority (84\%) of female sex in the 19 STAR mutation-carrying groups. The karyotype, $46, \mathrm{XY}$ and the ultrasound finding that shows no female internal genitalia in neonatal and infantile girls could aid in the clinical diagnosis of classic LCAH. The combination of these clinical clues, such as sex, external genitalia and family history, could help to determine the etiologies of non-syndromic biochemically uncharacterized PAI patients.

Recent studies have broadened the phenotypic spectrum of STAR defects (29), indicating that a minor subset shows later-onset PAI with relatively preserved mineralocorticoid- and testosterone-producing capacities (nonclassic LCAH). In this study, seven (patients 12-15, and 17-19) of the 19 patients with STAR mutations were diagnosed at two years of age or older, and five (patients 12-15, and 18) of the seven patients did not require mineralocorticoid replacement. Two $46, \mathrm{XY}$ boys (patient 13 and 14) of these seven 'late-onset' patients had normal male external genitalia. Further, none of the three evaluated patients (patients 12, 15 and 17) showed adrenal hyperplasia by ultrasound or computed tomography scan. Six (patients 12-15, 18 and 19) of the seven patients with nonclassic LCAH commonly harbored the p.Arg272Cys mutation, which retains about $28.4 \%$ of the activity of wild-type STAR in vitro (Noriyuki Katsumata, personal communication). Accordingly, we presume that the high residual activity of the p.Arg272Cys mutation caused the mild LCAH phenotype observed in the Japanese patients.

In some PAI patients with atypical phenotypes, the etiologies are difficult to diagnose on clinical grounds alone. One example from this study is one of the NNT mutation-carrying patients. Originally, NNT mutations were detected in a patient cohort of FGD, in which the patients retained their mineralocorticoid-producing capacity (13). In the present study, one patient (patient 47) with homozygous nonfunctional NNT mutation (p.Gln40Lysfs*9) likely had mild mineralocorticoid 
deficiency. This finding was consistent with that of recent studies that reported 22 patients with NNT mutationcarrying patients having mineralocorticoid deficiency $(21,30,31)$. The other example is AAAS mutationcarrying patients. We identified $A A A S$ mutations in siblings (family 46) that had been diagnosed as having non-syndromic ACTH resistance. We noticed that the siblings had subclinical alacrima after the identification of $A A A S$ mutations and affirmed the importance of research for alacrima in PAI patients. The observations, such as NNT defects with mineralocorticoid deficiency and atypical triple A syndrome, exemplify the clinical utility of mutation screening. By conducting whole exome sequencing, Chan et al. elucidated the unexpected genetic causes, such as defects of NROB1, CYP11A1, AIRE and $A A A S$, in 17 of the 43 patients with isolated or familial glucocorticoid deficiency (22). In this way, whole exome/ genome sequencing could be an alternative molecular method for genetic diagnosis.

We should mention the limitations of this study. First, we did not exclude autoimmune PAI by measuring 21-hydroxylase autoantibody, which is not available in Japan. However, this high frequency of genetic defects in our cohort would not decrease, even though we missed a few possible autoimmune PAI patients. Second, we did not conduct array CGH in all subjects and could not detect copy number variations.

In summary, we conducted genetic analyses in pediatric-onset biochemically uncharacterized PAI patients in Japan and identified genetic defects in 86\% of the subjects. Clinical characterization is an essential step for determining the possible etiologies, especially in cases of NROB1 defects and syndromic PAI such as typical triple A syndrome. However, in a subset of genetic defects, including nonclassic LCAH, NNT defects with mineralocorticoid deficiency and atypical triple A syndrome, it is difficult to presume the genetic causes based on the clinical findings alone. Molecular testing gives additional information for accurate diagnosis, which will enable more rational therapeutic approaches.

\section{Supplementary data}

This is linked to the online version of the paper at http://dx.doi.org/10.1530/ EJE-17-0027.

\section{Declaration of interest}

The authors declare that there is no conflict of interest that could be perceived as prejudicing the impartiality of this study.

\section{Funding}

This work was supported by a grant from the Ministry of Health, Labour and Welfare, Japan (Jitsuyoka Nanbyo-Ippan-014 to T Hasegawa) and grants from the Japan Society for the Promotion of Science (15K09599 and 16K09998).

\section{Author contribution statement}

T Hasegawa conceived the experiments. N Amano, S Narumi, M Hayashi, M Takagi, R Hachiya, G Sasaki and T Ishii performed the experiments. $\mathrm{K}$ Homma measured urine steroid metabolites of the patients. N Amano analyzed the data. $\mathrm{K}$ Imai and T Nakamura contributed for the detailed clinical data of patients with atypical NNT deficiency and atypical AAAS syndrome respectively. N Amano, S Narumi, T Ishii and T Hasegawa wrote the manuscript.

\section{Acknowledgements}

The authors thank Fumiko Kato for technical assistance, Prof Takao Takahashi for fruitful discussion and all physicians who referred patients. They also thank Noriyuki Katsumata for sharing the data of wild-type and p.R272C STAR activities in vitro.

\section{References}

1 New MI \& Levine LS. Recent advances in 21-hydroxylase deficiency. Annual Review of Medicine 198435 649-663. (doi:10.1146/annurev. me.35.020184.003245)

2 Simard J, Moisan AM \& Morel Y. Congenital adrenal hyperplasia due to 3beta-hydroxysteroid dehydrogenase/Delta(5)-Delta(4) isomerase deficiency. Seminars in Reproductive Medicine 200220 255-276. (doi:10.1055/s-2002-35373)

3 Homma K, Hasegawa T, Takeshita E, Watanabe K, Anzo M, Toyoura T, Jinno K, Ohashi T, Hamajima T, Takahashi Y et al. Elevated urine pregnanetriolone definitively establishes the diagnosis of classical 21-hydroxylase deficiency in term and preterm neonates. Journal of Clinical Endocrinology and Metabolism 200489 6087-6091. (doi:10.1210/jc.2004-0473)

4 Bose HS, Sugawara T, Strauss JF 3rd \& Miller WL. The pathophysiology and genetics of congenital lipoid adrenal hyperplasia. New England Journal of Medicine 1996335 1870-1878. (doi:10.1056/ NEJM199612193352503)

5 Katsumata N, Ohtake M, Hojo T, Ogawa E, Hara T, Sato N \& Tanaka T. Compound heterozygous mutations in the cholesterol sidechain cleavage enzyme gene (CYP11A) cause congenital adrenal insufficiency in humans. Journal of Clinical Endocrinology and Metabolism 200287 3808-3813. (doi:10.1210/jcem.87.8.8763)

6 Muscatelli F, Strom TM, Walker AP, Zanaria E, Recan D, Meindl A, Bardoni B, Guioli S, Zehetner G, Rabl W et al. Mutations in the DAX-1 gene give rise to both X-linked adrenal hypoplasia congenita and hypogonadotropic hypogonadism. Nature 1994372 672-676. (doi:10.1038/372672a0)

7 Suntharalingham JP, Buonocore F, Duncan AJ \& Achermann JC. DAX1 (NR0B1) and steroidogenic factor-1 (SF-1, NR5A1) in human disease. Best Practice and Research: Clinical Endocrinology and Metabolism 2015 29 607-619. (doi:10.1016/j.beem.2015.07.004)

8 Arboleda VA, Lee H, Parnaik R, Fleming A, Banerjee A, Ferraz-de-Souza B, Delot EC, Rodriguez-Fernandez IA, Braslavsky D, Bergada I et al. Mutations in the PCNA-binding domain of CDKN1C cause IMAGe syndrome. Nature Genetics 201244 788-792. (doi:10.1038/ng.2275)

9 Mandel H, Shemer R, Borochowitz ZU, Okopnik M, Knopf C, Indelman M, Drugan A, Tiosano D, Gershoni-Baruch R, 
Choder M et al. SERKAL syndrome: an autosomal-recessive disorder caused by a loss-of-function mutation in WNT4. American Journal of Human Genetics 200882 39-47. (doi:10.1016/j.ajhg.2007.08.005)

10 Narumi S, Amano N, Ishii T, Katsumata N, Muroya K, Adachi M, Toyoshima K, Tanaka Y, Fukuzawa R, Miyako K et al. SAMD9 mutations cause a novel multisystem disorder, MIRAGE syndrome, and are associated with loss of chromosome 7. Nature Genetics 2016 48 792-797. (doi:10.1038/ng.3569)

11 Weber A \& Clark AJ. Mutations of the ACTH receptor gene are only one cause of familial glucocorticoid deficiency. Human Molecular Genetics 19943 585-588. (doi:10.1093/hmg/3.4.585)

12 Metherell LA, Chapple JP, Cooray S, David A, Becker C, Ruschendorf F, Naville D, Begeot M, Khoo B, Nurnberg P et al. Mutations in MRAP, encoding a new interacting partner of the ACTH receptor, cause familial glucocorticoid deficiency type 2. Nature Genetics 200537 166-170. (doi:10.1038/ng1501)

13 Meimaridou E, Kowalczyk J, Guasti L, Hughes CR, Wagner F, Frommolt P, Nurnberg P, Mann NP, Banerjee R, Saka HN et al. Mutations in NNT encoding nicotinamide nucleotide transhydrogenase cause familial glucocorticoid deficiency. Nature Genetics 201244 740-742. (doi:10.1038/ng.2299)

14 Prasad R, Chan LF, Hughes CR, Kaski JP, Kowalczyk JC, Savage MO, Peters CJ, Nathwani N, Clark AJ, Storr HL et al. Thioredoxin reductase 2 (TXNRD2) mutation associated with familial glucocorticoid deficiency (FGD). Journal of Clinical Endocrinology and Metabolism 2014 99 E1556-E1563. (doi:10.1210/jc.2013-3844)

15 Hughes CR, Guasti L, Meimaridou E, Chuang CH, Schimenti JC, King PJ, Costigan C, Clark AJ \& Metherell LA. MCM4 mutation causes adrenal failure, short stature, and natural killer cell deficiency in humans. Journal of Clinical Investigation 2012122 814-820. (doi:10.1172/JCI60224)

16 Handschug K, Sperling S, Yoon SJ, Hennig S, Clark AJ \& Huebner A. Triple A syndrome is caused by mutations in AAAS, a new WD-repeat protein gene. Human Molecular Genetics 2001 10 283-290. (doi:10.1093/hmg/10.3.283)

17 Heino M, Peterson P, Kudoh J, Shimizu N, Antonarakis SE, Scott HS \& Krohn K. APECED mutations in the autoimmune regulator (AIRE) gene. Human Mutations 200118 205-211. (doi:10.1002/humu.1176)

18 Mosser J, Lutz Y, Stoeckel ME, Sarde CO, Kretz C, Douar AM, Lopez J, Aubourg P \& Mandel JL. The gene responsible for adrenoleukodystrophy encodes a peroxisomal membrane protein. Human Molecular Genetics 19943 265-271. (doi:10.1093/hmg/3.2.265)

19 Fasano T, Pisciotta L, Bocchi L, Guardamagna O, Assandro P, Rabacchi C, Zanoni P, Filocamo M, Bertolini S \& Calandra S. Lysosomal lipase deficiency: molecular characterization of eleven patients with Wolman or cholesteryl ester storage disease. Molecular Genetics and Metabolism 2012105 450-456. (doi:10.1016/j.ymgme.2011.12.008)

20 Berendse K, Engelen M, Linthorst GE, van Trotsenburg AS \& Poll-The BT. High prevalence of primary adrenal insufficiency in Zellweger spectrum disorders. Orphanet Journal of Rare Diseases 20149133. (doi:10.1186/s13023-014-0133-5)

21 Guran T, Buonocore F, Saka N, Ozbek MN, Aycan Z, Bereket A, Bas F, Darcan S, Bideci A, Guven A et al. Rare causes of primary adrenal insufficiency: genetic and clinical characterization of a large nationwide cohort. Journal of Clinical Endocrinology and Metabolism 2016101 284-292. (doi:10.1210/jc.2015-3250)

22 Chan LF, Campbell DC, Novoselova TV, Clark AJ \& Metherell LA. Whole-exome sequencing in the differential diagnosis of primary adrenal insufficiency in children. Frontiers in Endocrinology 20156 113. (doi:10.3389/fendo.2015.00113)

23 Amano N, Naoaki H, Ishii T, Narumi S, Hachiya R, Nishimura G $\&$ Hasegawa T. Radiological evolution in IMAGe association: a case report. American Journal of Medical Genetics Part A 2008 146a 2130-2133. (doi:10.1002/ajmg.a.32425)

24 Mitsui T, Narumi S, Inokuchi M, Nagasaki K, Nakazawa M, Sasaki G $\&$ Hasegawa T. Comprehensive next-generation sequencing analyses of hypoparathyroidism: identification of novel GCM2 mutations. Journal of Clinical Endocrinology and Metabolism 201499 E2421-E2428. (doi:10.1210/jc.2014-2174)

25 Takagi M, Narumi S, Asakura Y, Muroya K, Hasegawa Y, Adachi M \& Hasegawa T. A novel mutation in SOX2 causes hypogonadotropic hypogonadism with mild ocular malformation. Hormone Research in Paediatrics 201481 133-138. (doi:10.1159/000355279)

26 Huang Z, Ye J, Han L, Qiu W, Zhang H, Yu Y, Liang L, Gong Z \& Gu X. Identification of five novel STAR variants in ten Chinese patients with congenital lipoid adrenal hyperplasia. Steroids 2016108 85-91. (doi:10.1016/j.steroids.2016.01.016)

27 Kim JM, Choi JH, Lee JH, Kim GH, Lee BH, Kim HS, Shin JH, Shin CH, Kim CJ, Yu J et al. High allele frequency of the p.Q258X mutation and identification of a novel mis-splicing mutation in the STAR gene in Korean patients with congenital lipoid adrenal hyperplasia. European Journal of Endocrinology 2011165 771-778. (doi:10.1530/EJE-11-0597)

28 Nakae J, Tajima T, Sugawara T, Arakane F, Hanaki K, Hotsubo $\mathrm{T}$, Igarashi $\mathrm{N}$, Igarashi $\mathrm{Y}$, Ishii $\mathrm{T}$, Koda $\mathrm{N}$ et al. Analysis of the steroidogenic acute regulatory protein (StAR) gene in Japanese patients with congenital lipoid adrenal hyperplasia. Human Molecular Genetics 19976 571-576. (doi:10.1093/hmg/6.4.571)

29 Metherell LA, Naville D, Halaby G, Begeot M, Huebner A, Nurnberg G, Nurnberg P, Green J, Tomlinson JW, Krone NP et al. Nonclassic lipoid congenital adrenal hyperplasia masquerading as familial glucocorticoid deficiency. Journal of Clinical Endocrinology and Metabolism 200994 3865-3871. (doi:10.1210/jc.2009-0467)

30 Roucher-Boulez F, Mallet-Motak D, Samara-Boustani D, Jilani H, Ladjouze A, Souchon PF, Simon D, Nivot S, Heinrichs C, Ronze $\mathrm{M}$ et al. NNT mutations: a cause of primary adrenal insufficiency, oxidative stress and extra-adrenal defects. European Journal of Endocrinology 2016175 73-84. (doi:10.1530/EJE-16-0056)

31 Weinberg-Shukron A, Abu-Libdeh A, Zhadeh F, Carmel L, Kogot-Levin A, Kamal L, Kanaan M, Zeligson S, Renbaum P, Levy-Lahad E et al. Combined mineralocorticoid and glucocorticoid deficiency is caused by a novel founder nicotinamide nucleotide transhydrogenase mutation that alters mitochondrial morphology and increases oxidative stress. Journal of Medical Genetics $2015 \mathbf{5 2}$ 636-641. (doi:10.1136/jmedgenet-2015-103078)
Received 13 January 2017

Revised version received 11 May 2017

Accepted 18 May 2017 